\title{
Pulsed neodymium-YAG laser trabeculotomy: energy requirements and replicability
}

\author{
GORDON NEALE DUTTON,' DONALD ALLAN, ${ }^{2}$ AND SOPHIA A CAMERON
}

From the 'Tennent Institute of Ophthalmology, Western Infirmary, University of Glasgow, Glasgow G11 6NT, and ${ }^{2}$ West of Scotland Health Boards, Department of Clinical Physics, and Bio-Engineering, Glasgow G4 9LF

SUMMARY Short pulsed laser trabeculotomy has been shown to reduce intraocular pressure in patients with primary open angle glaucoma. This study seeks to determine the energy levels required to produce a fistula into the canal of Schlemm for four different Q-switched neodymiumYAG lasers. The laser was fired at fixed human trabecular meshwork specimens at a range of energy settings for each laser and the characteristics and replicability of the lesions produced were analysed. Energy levels between 3 and $5 \mathrm{~mJ}$ were sufficient to produce fistulae into the canal of Schlemm with an approximately $50 \%$ success rate for each instrument.

The pulsed neodymium-YAG laser may be used in a variety of ways for the treatment of glaucoma. These have been recently reviewed ${ }^{\prime 2}$ and include peripheral iridotomy, reopening of trabeculotomy, graded trabeculectomy, lysis of synaechiae, cyclodialysis, sclerostomy, goniotomy, and internal trabeculotomy. Short pulsed lased internal trabeculotomy was first described by $\mathrm{Krasnov}^{3}$ as a means of treating glaucoma. Unfortunately the pressure lowering effects of his treatment were not sustained beyond a few months.

Short pulsed laser trabeculotomy has been performed on a variety of ape species. ${ }^{4-8}$ Depending on the type of instrument used, energy levels between $0.5^{5}$ and $305 \mathrm{~mJ}^{4}$ have been employed to produce a fistula into the canal of Schlemm. However, these studies have shown that there is little resultant effect on intraocular pressure or facility of outflow, and when the lesions heal they are covered by a sheet of endothelial cells with a Descemet-like basement membrane. It has, however, been argued that the failure to reduce normal intraocular pressure in a healthy monkey cannot be compared with the need to restore normal pressure in a patient with glaucoma. ${ }^{8}$ Moreover, a fistula through which there is a persistent flow of aqueous owing to a pressure differential would be more likely to remain patent.

Theoretical considerations suggest that 20 perforations of $10 \mu \mathrm{m}$ diameter between the anterior chamber and the canal of Schlemm should restore Correspondence to G N Dutton, MD. normal outflow facility in open angle glaucoma. ${ }^{9}$ For clinical studies the type of instrument used, the energy levels chosen, and the number of laser applications required to treat patients with glaucoma by pulsed laser trabeculotomy have hitherto been chosen empirically. ${ }^{30-12}$ When three to six superimposed laser applications were used, closure of the fistula ensued. ${ }^{12}$ However, multiple single laser applications have produced a sustained reduction in intraocular pressure. ${ }^{1011}$

Owing to microscopic variations in the morphological characteristics of the trabecular meshwork, lesions produced at the same laser energy settings are not entirely replicable, and it has been argued that extensive experimental trial and error methods with electron microscopic evaluation are required to produce data to guide future research. ${ }^{13}$ Most of the morphological studies of pulsed laser trabeculotomy have been carried out on lesions produced with specially built apparatus, and the energy levels used are not comparable with those required for instruments which are currently available.

We have recently described the aiming criteria quired to produce a fistula into the canal of uhlemm ${ }^{14}$ and have shown that lesions produced in the trabecular meshwork are to a certain extent energy dependent. ${ }^{15}$ Moreover, we have demonstrated that the energy levels required to produce a fistula in vivo are similar to those which were determined by in-vitro experiments. ${ }^{14}$

In order to establish guidelines for future clinical 
research we have extended our previous work and have carried out an in-vitro scanning electron microscopic study of short pulsed Nd-YAG laser trabeculotomy lesions, produced by four currently marketed instruments. The energy levels required to produce a fistula into the canal of Schlemm were ascertained, and the replicability of the lesions produced by one or two such energy levels for each instrument were determined.

\section{Materials and methods}

Four Q-switched pulsed Nd-YAG lasers were employed. They comprised the Biophysic Médical Nanolas laser using the $10^{\circ}$ convergence angle, the Zeiss Visulas laser, the Lasag Microruptor II, and the Lasag Topaz instruments.

Human trabecular tissue was prepared from donor eyes which had not suffered antecedent ocular disease. Tissues were fixed in $3 \%$ cacodylate buffered glutaraldehyde, and $2-3 \mathrm{~mm}$ wide radial segments of corneoscleral tissue containing the trabecular meshwork were prepared. The ciliary body and iris tissues were removed by sharp dissection from each specimen without disturbing the trabecular meshwork.

A small plastic waterbath was prepared, and a Trokel gonioscopic lens (Ocular Instruments Inc), designed for use with pulsed Nd-YAG lasers, was mounted with a watertight seal into one end. The bath was filled with normal saline. Each specimen was in turn mounted on cardboard and immersed in the bath in such a manner that the trabecular meshwork could be clearly seen with the laser slitlamp microscope through the centre of the Trokel lens.

Each laser was adjusted to the energy settings described, and the laser was aimed and fired twice at the posterior trabecular meshwork, close to the cut edge at each side of the wedge shaped specimen. This technique permitted subsequent scanning electron microscopic assessment of the lesions in both the transverse and vertical dimensions.

Each specimen was reimmersed in $0.2 \mathrm{M}$ cacodylate buffer and prepared for scanning electron microscopy (Jeol JSMT 2000) by critical point drying and gold coating. Each lesion was then photographed.

It was not possible to produce exact measurements of the size of each lesion owing to variations in shape and the difficulty in defining the boundaries of each lesion. Thus lesions were graded both for size and neatness according to the protocol below, in preference to using quantitative but imprecise measurements. Fig. 1 illustrates the morphological characteristics of each of these grades.

\section{GRADINGS OF ND-YAG LASER LESIONS}

\begin{tabular}{|c|c|}
\hline \multicolumn{2}{|c|}{ Size grade } \\
\hline 0 & No lesion \\
\hline 1 & Superficial trabecular compression or disruption \\
\hline 2 & $\begin{array}{l}\text { Hole in trabecular meshwork which does not enter the canal } \\
\text { of Schlemm }\end{array}$ \\
\hline 3 & $\begin{array}{l}\text { Small hole in trabecular meshwork which enters the canal of } \\
\text { Schlemm }\end{array}$ \\
\hline 4 & $\begin{array}{l}\text { Large hole in trabecular meshwork which enters the canal of } \\
\text { Schlemm with or without superficial damage to the outer } \\
\text { wall of the canal }\end{array}$ \\
\hline 5 & $\begin{array}{l}\text { Hole which perforates the outer wall of the canal of } \\
\text { Schlemm }\end{array}$ \\
\hline \multicolumn{2}{|c|}{ Neatness grade } \\
\hline A & Smooth m \\
\hline B & Ragged margins to hole. \\
\hline
\end{tabular}

Lesions were independently graded by three observers. The score sheets were collated, the interobserver repeatability assessed, and overall grades were assigned for size and neatness. These were accorded as follows:
Size grade
(a) If unanimous - that grade
(b) If 2 out of 3 agreed - the majority grade
(c) If all 3 differed - the central grade

\section{Neatness grade}

(a) If unanimous - that grade

(b) If not unanimous, the majority decision.

Two experiments were carried out for each of the four lasers. First, between three and six shots were fired at the posterior trabecular meshwork of separate specimens for each setting in a range of energy settings, and the lesions produced were graded. Energy levels required to produce reliably a fistula into the canal of Schlemm without disrupting the outer wall were determined. For the second experiment multiple shots were fired at such an energy level and the lesions produced were assessed in the same manner.

\section{Results}

Fig. 1 illustrates the appearances of the gradings described above.

The size gradings assigned by the three assessors for all micrographs were compared. For the 202 micrographs studied $86 \%$ of gradings showed at least two out of three in agreement, while for $14 \%$ the assessments differed. The $\chi^{2}$-test was employed to verify that the level of concordance for the gradings was uniform for individual experiments. There was no significant difference $(0 \cdot 2<\mathrm{p}<0 \cdot 5)$. The neatness gradings demonstrated $40 \%$ complete concordance and $60 \%$ showed two out of three in agreement. The $\chi^{2}$-test again confirmed that there was no significant variation between experiments $(p<0 \cdot 5)$. These figures indicate that both the gradings and the subjective assessments of neatness employed give satisfactory consistency. 


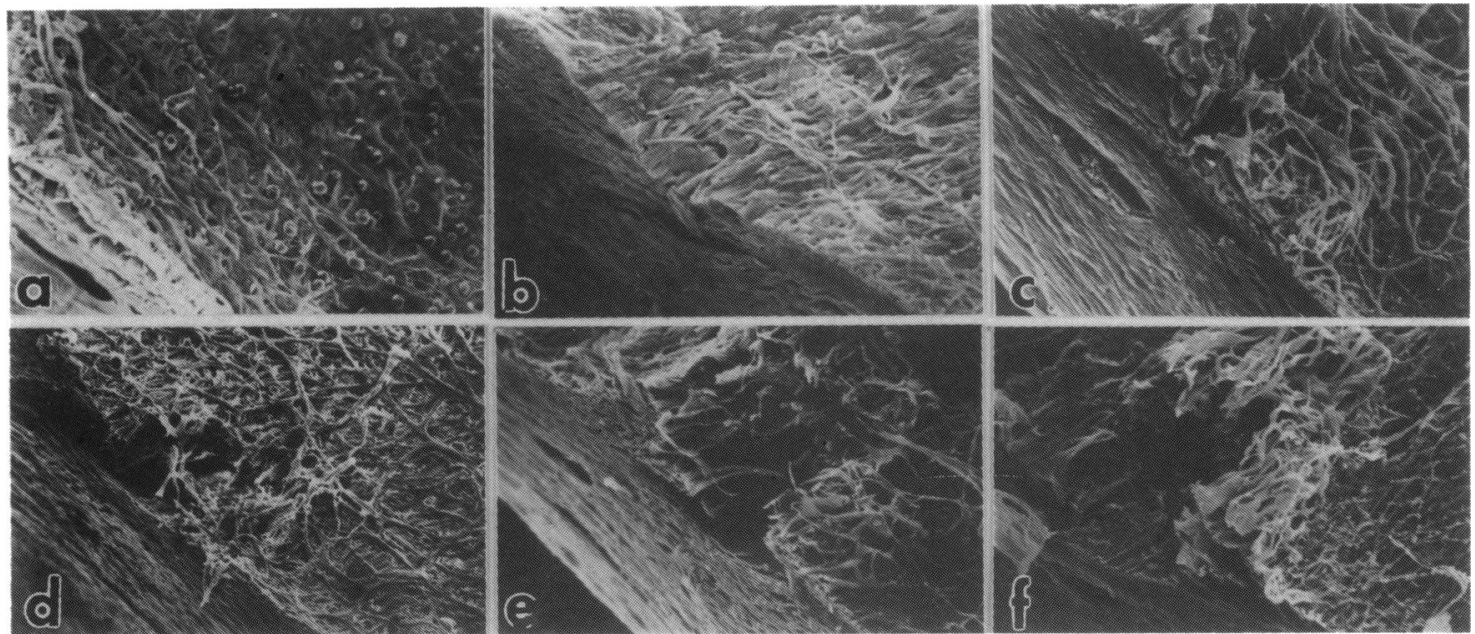

Fig. 1 Scanning electron micrographs of lesions produced in the trabecular meshwork by single $Q$-switched neodymiumYAG laser pulses graded according to the protocol described. a: Grade 0. b: Grade 1. c: Grade 2. d: Grade 3. e: Grade 4.

f: Grade 5. $\times 170$.

Figs. 2-5 provide a graphical analysis of the grades of the lesions produced in the trabecular meshwork in relation to the energy levels applied with each instrument. The graphs on the left relate to the extent of damage produced by each energy level employed while the graphs on the right provide illustrations of the replicability of the lesions produced for single energy settings. It can be seen that there is no strong correlation between the energy applied and the severity of damage produced. For the Biophysic Médical Nanolas, the Zeiss Visulas, and the Lasag Topaz apparatus grade 4 lesions were produced only with pulses of $3 \mathrm{~mJ}$ or more, whereas with the Microruptor II lower energies sufficed to produce such a degree of damage. Apart from this caveat the relationship of the energy levels employed to the lesions produced did not differ in any consistent manner between instruments.

There was no difference between the instruments with respect to the 'neatness' of the lesions produced. Approximately half the lesions were graded as neat and half as ragged for each instrument.

With regard to the replicability of the lesions produced, the likelihood of producing a fistula into the canal of Schlemm with the energy levels selected approximated to $50 \%$, with a slightly higher success rate for the Zeiss Visulas instrument.

\section{Discussion}

Before applying short pulsed laser lesions on the trabecular meshwork with the intention of treating glaucoma it is important to establish the energy requirements to produce a patent fistula into the canal of Schlemm without disruption of the outer wall of the canal. Although these requirements have been established for specially built apparatus ${ }^{1617}$ there have been few studies aimed at delineating the energy requirements for commercially available apparatus. ${ }^{15}$ Criticisms of the above study include the fact that the tissue was fixed and not fresh, and that the optical system of a waterbath differs from that of the human eye. However, we have recently conducted a study with our own specially built apparatus on patients whose eyes were subsequently enucleated for melanoma and have found that the energy settings which were determined in vitro with fixed tissue ${ }^{16} 17$ did not differ significantly from those required to produce a fistula into the canal of Schlemm in vivo. ${ }^{14}$ Moreover, the alternative primate experiments are difficult and expensive, and the different morphology of the trabecular meshwork makes the results difficult to interpret and extrapolate for use in man. The disparity in the grading assessments apportioned by the three authors in the present study arose as a result of the difficulties in definitively interpreting the degree of tissue disruption. Nevertheless, the results obtained indicated that, although the extent of tissue damage is partly energy dependent, there is considerable variation between shots at the same energy levels.

The instruments which were investigated differ considerably both in design and construction and in price. The features most likely to be potential causes of differing performance of the lasers are pulse duration ( $7 \mathrm{~ns}$ for Visulas, $5 \mathrm{~ns}$ for Nanolas, and $12 \mathrm{~ns}$ for Topaz and Microruptor II), beam convergence angle $\left(12^{\circ}\right.$ for Visulas, $10^{\circ}$ selected for the Nanolas, ${ }^{15}$ 


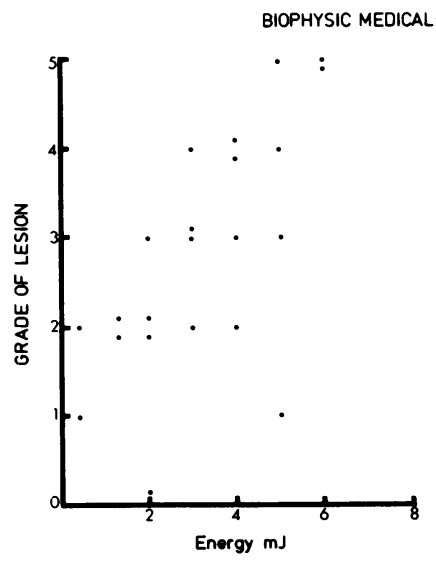

Fig. 2 Graphs relating the grades of the lesion produced in the trabecular meshwork in relation to the laser energy applied with the Biophysic Médical Nanolas laser. The graph on the left illustrates the results obtained for a range of energy settings and the graph on the right illustrates the replicability of the lesions produced at a single energy setting.
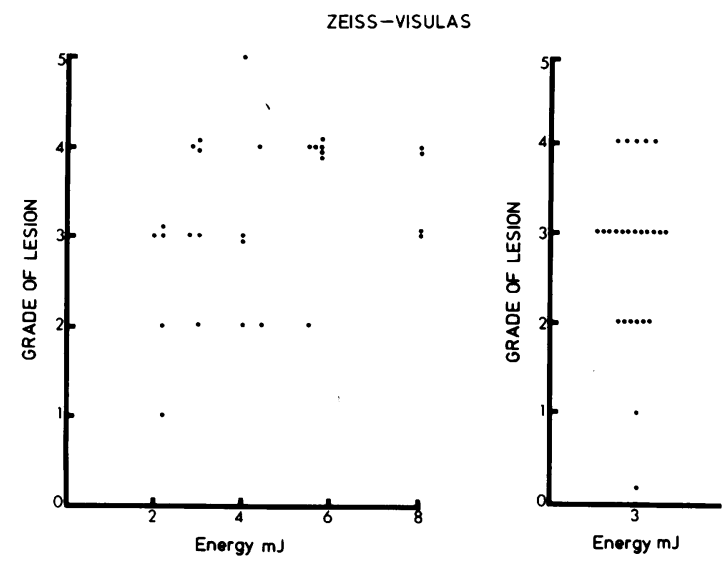

Fig. 3 Graphs relating the grades of the lesion produced in the trabecular meshwork in relation to the laser energy applied with the Zeiss Visulas laser. The graph on the left illustrates the results obtained for a range of energy settings and the graph on the right illustrates the replicability of the lesions produced at a single energy setting.

and $16^{\circ}$ for Topaz and Microruptor II), and the transverse mode structure of the laser beam (low order mode for Visulas and Nanolas and $\mathrm{TEM}_{00}$ for Topaz and Microruptor II). All figures are from manufacturers' specifications.

Furthermore, the method of controlling the laser pulse energy may affect the stability of mode structure with respect to pulse energy. In both the Visulas and Nanolas equipment the laser source operates at a constant energy setting and the

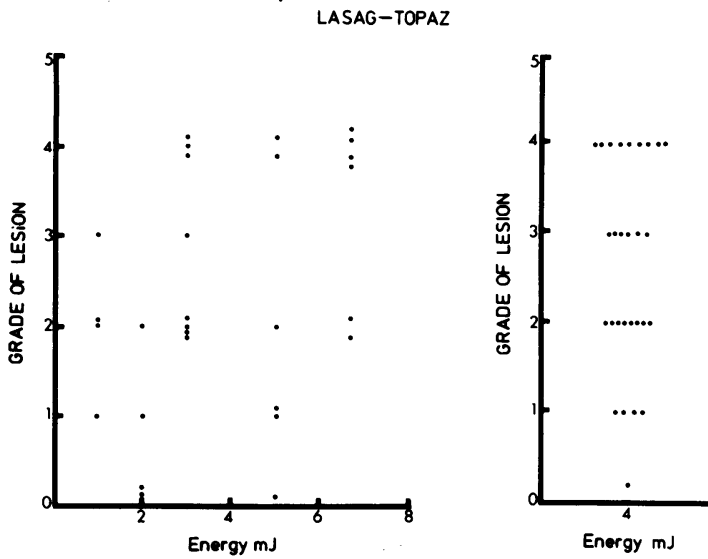

Fig. 4 Graphs relating the grades of the lesion produced in the trabecular meshwork in relation to the laser energy applied with the Lasag Topaz laser. The graph on the left illustrates the results obtained for a range of energy settings and the graph on the right illustrates the replicability of the lesions produced at a single energy setting.

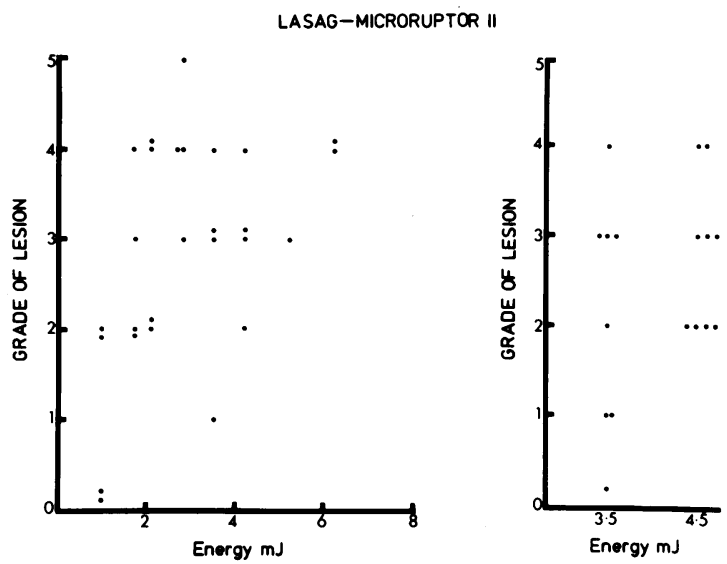

Fig. 5 Graphs relating the grades of the lesion produced in the trabecular meshwork in relation to the laser energy applied with the Lasag Microruptor II. The graph on the lefi illustrates the results obtained for a range of energy settings and the graph on the right illustrates the replicability of the lesions produced at two energy settings.

delivered output is varied by adjustable attenuators, while for the Topaz and Microruptor II the energy level of the laser itself is varied.

In practice, however, there seems to be little to choose between the four lasers. The tendency of the Microruptor II to produce grade 4 lesions with slightly lower pulse energy than the other instruments may result from the more pure mode structure giving a slightly better concentration of laser energy at the point of focus. This would cause more efficient 
plasma production and more effective tissue disruption. Although the Lasag Topaz is nominally similar in characteristics, the lesions produced with it do not show such an effect.

Clinical studies have indicated that reduction in intraocular pressure can be produced in patients with chronic open angle glaucoma by means of shortpulsed laser trabeculotomy..$^{3011}$ However, the reduction in intraocular pressure may not be sustained, perhaps on account of healing which occludes the tiny fistulae. Neat fistulae into the canal of Schlemm which do not become occluded owing either to reepithelialisation or to healing of the damaged outer wall of the canal would be the ideal goal. To this end the aiming criteria have recently been established ${ }^{14}$ (the beam must be aimed at the posterior trabecular meshwork through the centre of the gonioscopic mirror), while the results described here suggest that pulse energies of between 3 and $5 \mathrm{~mJ}$ should be employed. A success rate of about $50 \%$ for the creation of a fistula into the canal of Schlemm might then be attained. Further experimental clinical studies are required to establish whether this technique is effective in practice.

The authors thank the staff of Bristol Eye Hospital, the Eye Department of Ninewells Hospital, Dundee, the Royal Alexandra Infirmary, Paisley, and Keeler Ltd for permission to carry out experimental work with their instruments. This work was supported by a grant (No. 721) from the Scottish Hospital Endowments Research Trust.

\section{References}

1 Klapper RM. The role of neodymium: Yag laser in microsurgery of the glaucomas. Int Ophthalmol 1985; 25: 101-17.

2 Cohn H, Aron B, Dhermy P, Aron-Rosa D. Laser YAG et traitement des glaucomes. Etude experimentale et indications pratiques. Bull Soc Ophthalmol Fr 1985; 85: 939-41.
3 Krasnov MM. Q-switched laser goniopuncture. Arch Ophthalmol 1974: 92: 37-41.

4 Van der Zypen E, Fankhauser F. The ultrastructural features of laser trabeculopuncture and cyclodialysis. Problems related to successful treatment of chronic simple glaucoma. Ophthalmologica 1979; 179: 189-200.

5 Ticho U, Mahler J, Sekeles E, et al. Low-energy laser trabeculotomies in primates. Exp Eye Res 1981; 33: 11-8.

6 Bonney CH, Gaasterland DE, Rodrigues MM, Raymond JJ, Donohoo P. Short-term effects of Q-switch ruby laser on monkey anterior chamber angle. Invest Ophthalmol Vis Sci 1982; 22: 310-8.

7 Gaasterland DE, Bonney $\mathrm{CH}$, Rodrigues MM, Kuwabara T. Long-term effects of Q-switched ruby laser on monkey anterior chamber angle. Invest Ophthalmol Vis Sci 1985; 26: 129-35.

8 Melamed S, Pei J, Puliafito CA, Epstein DL. Q-switched neodymium YAG laser trabeculopuncture in monkeys. Arch Ophthalmol 1985; 103: 129-33.

9 Goldschmidt CR, Ticho U. Theoretical approach to laser trabeculotomy. Med Phys 1978; 5: 92-9.

10 Robin AL, Pollack IP. The Q-switched ruby laser in glaucoma. Ophthalmology 1984; 91: 366-72.

11 Robin AL, Pollack IP. Q-switched neodymium-YAG laser angle surgery in open-angle glaucoma. Arch Ophthalmol 1985; 103: 793-5.

12 Epstein DL, Melamed S, Puliafito CA, Steinert RF. Neodymium: YAG laser trabeculopuncture in open-angle glaucoma. Ophthalmology 1985; 92: 931-7.

13 Van der Zypen E, Bebie H, Fankhauser F. Morphological studies about the efficiency of laser beams upon the structures of the angle of the anterior chamber. Facts and concepts related to the treatment of chronic simple glaucoma. Int Ophthalmol 1979; 1, 2: 109-22.

14 Dutton GN, Allan D, Cameron SA. Aiming criteria for neodymium-YAG laser trabeculotomy a clinico-pathological study. Eye 1987; 1: 401-5.

15 Dutton GN, Cameron SA, Allan D, Thomas R. Parameters for neodymium-YAG laser trabeculotomy: an in-vitro study. $\mathrm{Br} J$ Ophthalmol 1987; 71 : 782-6.

16 Venkatesh S, Guthrie S, Foulds WS, Lee WR, Cruickshank FR, Bailey RT. In vitro studies with a pulsed neodymium-YAG laser. Br J Ophthalmol 1985; 69: 86-91.

17 Venkatesh S, Lee WR, Guthrie S, et al. An in-vitro morphological study of Q-switched neodymium-YAG laser trabeculotomy. Br J Ophthalmol 1986; 70: 89-96.

Accepted for publication 31 March 1988. 